\title{
Correction to: Weighted Upper Edge Cover: Complexity and Approximability
}

Kaveh Khoshkhah, Mehdi Khosravian Ghadikolaei, Jérôme Monnot, and Florian Sikora

\section{Correction to: \\ Chapter "Weighted Upper Edge Cover: Complexity and Approximability" in: G. K. Das et al. (Eds.): \\ WALCOM: Algorithms and Computation, LNCS 11355, https://doi.org/10.1007/978-3-030-10564-8_19}

Corollary 13 was found to be wrong and, therefore, it and everything pertaining to Corollary 13 was removed from the chapter. 